\title{
Potential Flow Simulation through Lagrangian Interpolation Meshless Method Coding
}

\author{
T. Avudaiappan ${ }^{1 \dagger}$, V. Vijayan ${ }^{1}$, S. Sundara Pandiyan $^{2}$, M. Saravanan ${ }^{3}$ and S. Dinesh ${ }^{1}$ \\ ${ }^{1}$ K. Ramakrishnan College of Technology, Trichy, Tamilnadu, India \\ ${ }^{2}$ Christ (Deemed to be University), Bengaluru, Karnataka, India \\ ${ }^{3}$ SRM Institute of Science and Technology, Kattankulathur, Tamilnadu, India \\ †Corresponding Author Email: avudaiappanmecse@gmail.com
}

(Received May 15, 2018; accepted July 18, 2018)

\begin{abstract}
From the past many decades, mesh generation posed many challenges in the field of computational sciences for many researches. High rise in computational power has enabled many researches to tackle the problems of complex geometries. Due to the high need of computational power, computational cost also increased abruptly. In today's world, many academic and industry researches are willing to increase the use of present simulation technology; mesh generation plays a vital role in this aspect. we can say that many real-world simulation problems are dependent on mesh generation which has more chances in giving an inaccurate simulation results. In order to make the simulation process simpler, Meshless methods are introduced to the field of Computational Fluid Dynamics. This technique requires a less computational power compared to the computational power needed for generating the mesh. In the present dissertation, our main objective is to develop a scheme for Meshless method for the field of Computational Fluid Dynamics for flow over a blunt body. The performance of the present scheme is evaluated by comparing the simulation results with existing experimental data and also compared with the results obtained by generation of mesh using commercial CFD software.
\end{abstract}

Keywords: Lagrangian interpolation; Meshless coding; Flow simulation.

\section{INTRODUCTION}

The basic requirement for discrediting the given problem by Meshless schemes is by getting high reliability and computational efficiency. The satisfaction of these two factors can show the success of Meshless technique. Even though the basic ideas about Meshless schemes are clearly understood, developing an efficient Meshless method is a quite challenging task. Many factors like numerical integration, interpolation scheme and the schemes for calculating the boundary conditions should be clearly developed for an efficient Meshless scheme as shown in Figure 1.

This method was named as Smoothed Particle Hydrodynamics. This approach uses a particlebased approach with a technique by keeping the moving particles in the place of fluid. Then, the necessary governing equations are transformed into the required integral forms. Later, with the success of the smoothed particle Hydrodynamics, many researches increased the usage of Meshless methods and increased its applications. Therefore, Meshless methods was started its identity in many other fields in late $90 \mathrm{~s}$. Based on the excellent performance by Meshless methods, many attempts were made to increase its accuracy and consistency for increasing the accuracy of the simulation results (Atluri et. al, 2000)

Here $\Omega$ is the domain for which the kernel approximations are invoked. The computational model shown here is a circular model. This is solved using SPH approach. After modifying this scheme to many applications, Smoothed Particle Hydrodynamics increased its applications to wide range of problems. Many cases with magneto hydrodynamics, heat conduction, explosion phenomenon, free surface impacts were analyzed with scattering of cloud of grid points. (Jiun-Shyan, et.al, 2009). These SPH methods used an approach by invoking the kernel approximations for $\mathrm{u}(\mathrm{x})$ on the given domain. After the success of smoothed particle hydrodynamics, some small changes are invoked into its scheme for increasing the accuracy and consistency. With these changes, this scheme was renamed as Corrected smoothed partial hydrodynamics. 
The main motivation behind developing Meshless scheme was to escape from the burden of mesh. As many academic and industrial researchers globally are trying to simulate the realistic problems, today's simulation world needs to be user friendly to provide accurate results within the time (Lakshmanaprabu et al. (2017)). Therefore, to decrease the computational power and time, Meshless methods were developed for different kind of problems. This thesis is mainly concentrating on the potential flow analysis over a blunt body. As mesh generation plays a vital role in today's simulation world, Simulation world need a field expertise to generate the mesh. Also, a small error or fault in generation mesh may result into bad simulation results. In the given time period, for complex geometries, a very high computational power and time with field experts should undergo the mesh generation. Therefore, to escape from this burden, Meshless methods were developed. As this uses a simple technique by scattering a cloud of grid points, the complex level of solving the problem will be decreased and also the man-hours needed for solving the problem will also be decreased. In this dissertation, potential flow is simulated numerically over a blunt body problem in Computational Fluid Dynamics. The success of this dissertation will also serve as a motivation for many researchers to develop Meshless schemes to various other problems in the field of Computational Fluid Dynamics.
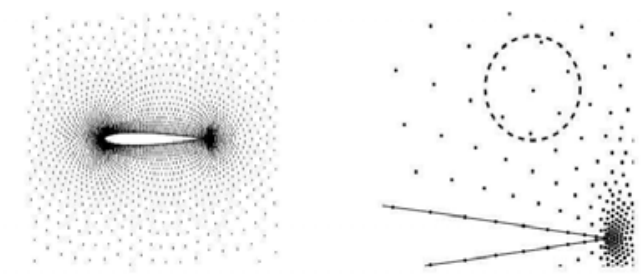

Fig. 1. Meshless kind of discretization for analyzing the flow over an airfoil

The idea of Meshless scheme came into mind when the existing discretization methods were not found to the much efficient. Initially, the motivation in developing the Meshless scheme was to simulate various applications from fields like geophysics, meteorology and geodesy. Then, the research in this area was developed in a systematically manner which are able to find the numerical solutions of Partial differential equations etc. The two basic Meshless schemes which were developed initially are moving least square methods and radial basis functions. Independently, Meshless scheme (local regression) was into use from the past 100 years. This high interest in Meshless schemes developed to exist in various flavors. In today's Computational Fluid Dynamics world, Meshfree methods applications are a bit rare in computational nanotechnology.

Mulay (2009) from Nanyang Technological University presented a brief knowledge on Meshless methods through a book titled: Meshless Methods and Their Numerical Properties. In this book, they clarified many queries related to
Meshless methods. This work was recognized for many numerical computational problems of many engineering problems. In this book, a detailed mathematical and analytical formulations for the most existing and developing methods in the field of Meshless methods. The authors of the book also concentrated on many numerical properties for Meshless methods like stability, adaptively, convergence, consistency etc. For the beginners to the Meshless methods, this book helps a lot in providing a strong fundamental base. (Mulay, et al. 2009 \& Babuska et al. 2002, 2010).

Even though many Analytical and Numerical scheme are into existence in today simulation world, Meshfree schemes are into much interest in scientific and engineering communities. The only reason behind this success is the lack of connectivity between grid points. The complexity in finite volume, finite element and finite difference methods in simulating complex geometries increased the increased the interest in developing Meshfree schemes. Therefore, the knowledge provided by Liu in his books on Meshless methods made many people globally to understand its algorithms, implementations and coding on many problems.

Later, to provide the information for developments on Meshless methods, Computational Methods on Applied Sciences published a book named, "Progress on Meshless Methods", which was written by Kansa EJ, Ferreira AJM, Fasshauer GE. This book's main objective is to explore the research field of Meshless methods. This book mainly concentrated on various Meshfree approximation techniques and their developments. These Meshfree approximation techniques are very much into interest in the today's research areas of computational mechanics (Francisco et al. 2009)

Compared to past, Meshfree schemes are increasing its considerations in various engineering applications and applied mathematics. Therefore, the above-mentioned book presents many works which addressed many engineering problems and also the contributions explored in the Meshfree methods held at Porto in the year 2007. This really helps a lot for many students, academicians and researchers interested in Meshless methods and computational mechanics. From the past many decades, Meshfree schemes have created its own impression in the world of computational fluid dynamics for solving various numerical techniques in the field of science and engineering. To explore its value, many meetings. After having a look at the above image, the first quire we get is about PU. Therefore, lets discuss about the PU initially which is most important in understanding the MM's. (Kaljevic, et al., 1997)

In solving the PDE's numerically, consistency, stability and convergence are the most important factors that need to be taken into consideration. The consistency requirements depend on the obtained PDE's level of order. For example, in a given problem, approximations for PDE with a Galerkin methods are of order of 31, then the solving of those 
PDE's requires a shape and test functions with a consistency level of 1-th order. If the condition in eq. 3.1 is satisfied, then the consistency order is obtained the given set of functions $\{\Phi\}$. (Unther 1998).

$$
\Sigma \quad \Phi_{\mathrm{i}}(\mathrm{x}) \mathrm{p}\left(\mathrm{x}_{\mathrm{i}}\right)=\mathrm{p}(\mathrm{x})
$$

In the above expression, $\Phi$ represents the PU (Partition of unity). On the basis of determined PU, approximations will be done for the given problem. Based on the determined PU's, a mesh based, or Meshless scheme kind of grid can be easily generated. The above figure shows the different classifications of Mesh based methods and the classification of Meshless methods. Therefore, this section describes the classifications of Meshless schemes which are mentioned in the above representation. (Aluru 2000, Atluri et al. 1998, 1999, 2002).

\section{METHODOLOGY METHODS FOR FLOW OVER A BLUNT BODY}

Initially, the model for which Meshless scheme to used should be known. The code should be developed to create a model, or the code should have an option to import the model from a specific location. The imported model or the created model should be checked for errors. This means, geometry errors should not be found. In this project, we have considered a 2-D cylinder as a blunt body for the analysis.

After designing or importing the geometry, a cloud of grid points should be implemented in the geometry. If the problem taken is to simulate the flow externally, then the cloud of grid points should be implemented outside the body in the domain created. If the problem taken is to simulate the flow inside the body, the cloud of grid points should be generated inside the domain. These points can be generated randomly or by any forced source. The developed code should have an option to increase or decrease the generated grid points based on the accuracy obtained by computing the flow. Generating the cloud of grid points should be dependent of each other. If the Meshless schemes are implemented for complex geometries, random generation or random distribution of points will create a problem. For simple geometries, cloud of grid points can be generated randomly. The below Figure 1 represents the scattered cloud of grid points on the inner and outer boundary of the domain along with 1000 points inside the domain. This figure is a very course scattering of grid points. The below figure represents the Meshless technique with 10,000 points in the domain. In this representation, there are no boundary points. With these grid points, one can easily capture the flow over the blunt body.

In this work, the developed code simulates the flow internally of a blunt body and capture the eddies formed. The code has 10,000 grid points inside the body and 100 grid points on the boundary of the body. As this code is for a 2-D cylinder, the grid points generated are sufficient to compute the required flow. As the grid points are generated, we have to find the nearest points to each point for finding the Lagrangian co-efficient for determining the diffusion terms for implementing the required equations. Therefore, for this purpose, we have to find the distance from each point to all other points to determine the neighboring points. As we are having the $\mathrm{x}$ and $\mathrm{y}$ coordinates for each point in the grid, the developed code should be able to determine the distances between the points. In the code developed in this work, it is determining the distances between the points inside the grid and also on the boundaries of the body. These distances are will be used in further steps which are going to be discussed below.

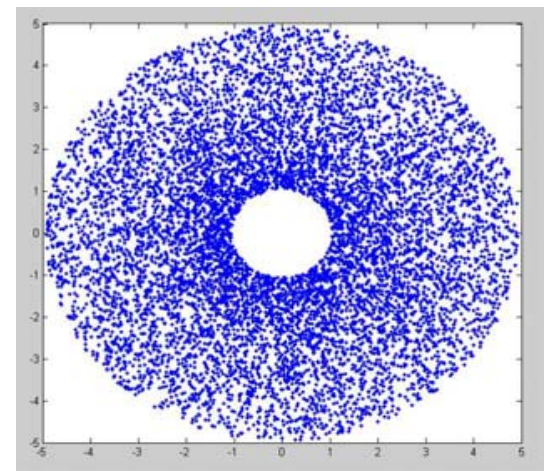

Fig. 1. Meshless techniques with 10,000 points in the domain

As the distances from each point are known, it is very easy to find the nearest points for each point in the grid. This can be done in many ways. It can be done by sorting the distances based on maximum and minimum, it can also be done by displaying the first five distances after zero as shown in Figure 2. There are many ways to determine the nearest points.

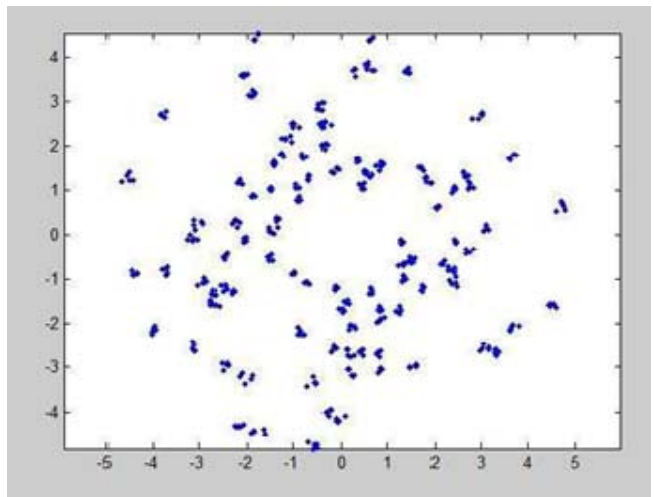

Fig. 2. Nearest points from each point in the domain

In Figure 3, nearest points are named as npstore in the developed code. For example, from figure 3, for 1st point nearest points are 108, 35, 105, and 63,101. After generating these nearest points, random points are seeded in the present thesis so that, at every execution of the code, random function generates the points at same axis positions. In the code developed in this thesis, we used sorting tool for finding the nearest grid points from each point. It calculates the 
distances from each point and then filters the first five minimum distances. Even though sorting tool is computationally costly, this code is developed using that to decrease the complexity of the work. When a Meshless scheme is developed for a more complex geometry, another method for determining the nearest points should be developed to decrease the computational cost and power.

$\begin{array}{rrrrrr}\text { npstore }= & & & & & \\ 1 & 108 & 35 & 105 & 63 & 101 \\ 2 & 108 & 35 & 63 & 105 & 113 \\ 3 & 108 & 35 & 63 & 113 & 101 \\ 4 & 63 & 108 & 113 & 35 & 101 \\ 5 & 113 & 63 & 108 & 35 & 101 \\ 6 & 113 & 63 & 108 & 35 & 75 \\ 7 & 113 & 75 & 63 & 108 & 35 \\ 8 & 113 & 75 & 117 & 45 & 63 \\ 9 & 75 & 117 & 45 & 113 & 63 \\ 10 & 117 & 75 & 45 & 113 & 106 \\ 11 & 117 & 45 & 75 & 106 & 40 \\ 12 & 117 & 45 & 106 & 75 & 40 \\ 13 & 45 & 117 & 106 & 40 & 78 \\ 14 & 106 & 45 & 117 & 40 & 92 \\ 15 & 106 & 40 & 45 & 117 & 58 \\ 16 & 106 & 40 & 58 & 45 & 69 \\ 17 & 58 & 106 & 40 & 69 & 92 \\ 18 & 58 & 69 & 106 & 107 & 40 \\ 19 & 58 & 107 & 61 & 69 & 106 \\ 210 & 107 & 61 & 58 & 87 & 69\end{array}$

Fig. 3. Representation of nearest points from 1 to 20

Till here, the present code creates a model, then generates a scattered cloud of points and determines the distances between the points and filters the nearest five points. Now, the nearest points are found for determining the diffusion terms for each point. Therefore, for determining the diffusive terms, Lagrangian coefficient for each point is found. Those Lagrangian coefficients are implemented in the required equations. In the developed code, Lagrangian coefficients are determined using Gauss-Sedal method. As the Lagrangian coefficients do not change at every time step and are independent, Lagrangian coefficients help us in connecting all the grid points from outer boundary to inner boundary grid points. These Lagrangian coefficients play a very vital role in Meshfree schemes. In this code, Lagrangian coefficients are found with respect to $\mathrm{x}$ and $\mathrm{y}$ for single differential and double differential as well. Therefore, for implementing the boundary conditions, Lagrangian coefficients help us in connecting for predicting the flow properties.

\section{FLUID DYNAMICS SIMULATION RESULTS}

As per the above discussed methodology for Meshless methods, velocity parameters were determined at each node in the grid. The found velocities were plotted as contours using postprocessing software Techplot 360 . Techplot 360 is software which renders and helps us to visualize contours, vectors, streamlines etc obtained after performing the simulations in any CFD software.

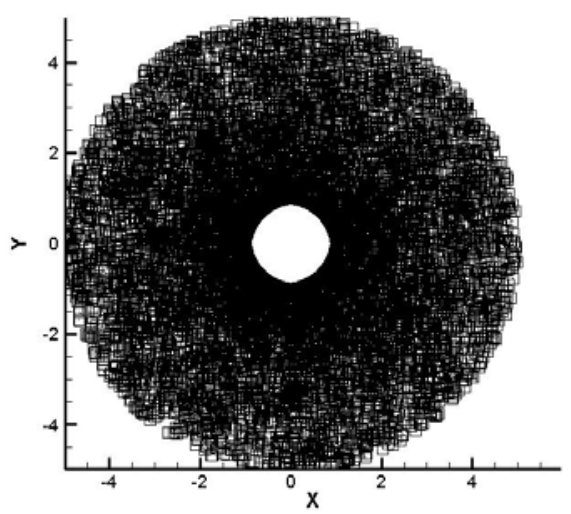

Fig. 4. Scattered Grid points in Techplot

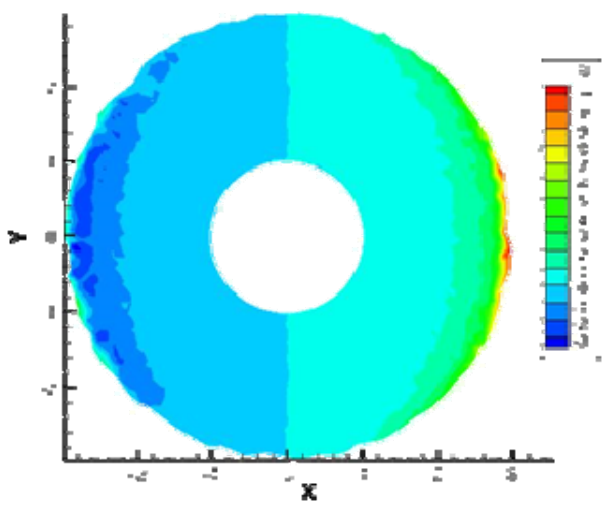

Fig. 5. Velocity Contours

The above Figure 4 shows the representation of scattered cloud of grid points visualized in Techplot 360, a post - processing CFD software. The above figure represents a fine Meshless discretization. With this discretization, behavior of the flow can be easily predicted.

The above figure represents the velocity contours visualized using Techplot 360 , CFD post processing software. These velocity contours are rendered by implementing the Meshless technique. This figure represents that Meshless scheme was able to capture the velocity contours at every grid point in the flow. After determining the velocity parameters at each grid point in the domain through the developed code, all the velocity parameters are stored in a separate variable and invoked into the Techplot 360 software according to its format. This present velocity contours represent the potential flow equation to the inner grid points. Later, this code can be developed by implementing the NavierStokes equations on the flow which can help in capturing eddy formed. For executing the code for determining the velocity parameters, time consumed was around 2 minutes by a normal computer.

\section{VALIDATIONS VERIFICATIONS}

AND

The above Figure 6 represents the velocity magnitude contours for flow over the blunt body. 
The above CFD analysis is performed for an inviscid flow with velocity $1 \mathrm{~m} / \mathrm{s}$. Flow is entering the domain through velocity inlet and outlet is defined as pressure outlet.

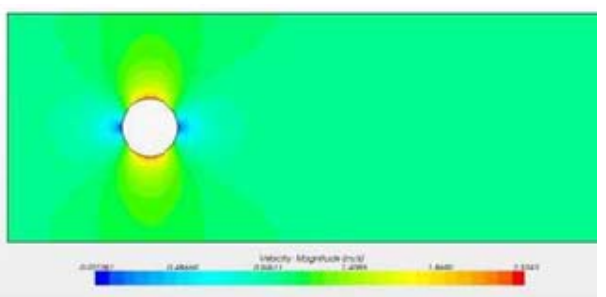

Fig. 6. Velocity magnitude Contours plot

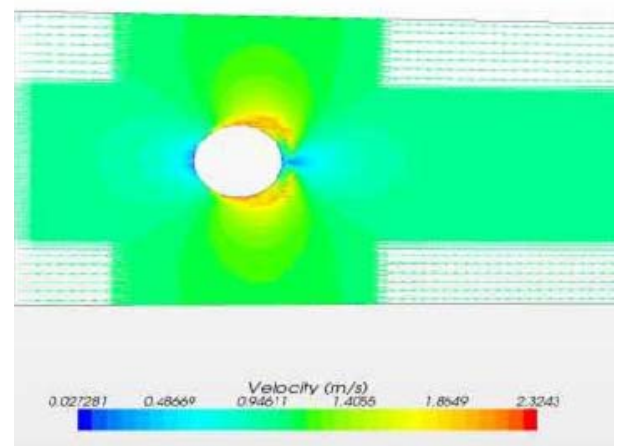

Fig. 7. Velocity Vectors for flow over a blunt body

The above figure 7 represents the velocity magnitude vectors for flow over the blunt body. From the above CFD analysis performed, velocity vectors are in the direction of $\mathrm{x}$ axis in the domain and due to the circular section in the domain; vectors are passing from the surface of the cylinder towards pressure outlet.

The above figure 8 represents the velocity contours obtained through Matlab inbuilt. This analysis is performed with velocity $1 \mathrm{~m} / \mathrm{s}$. In this Matlab code, potential flow is solved by implementing the mesh. With the above CFD analysis performed using the Meshless technique developed in this thesis, we have validated the results with the contour plots obtained through STAR-CCM+ and Matlab inbuilt codes. Meshless code was successfully able to determine and capture the velocity vectors at each node. Velocity vectors obtained by implementing

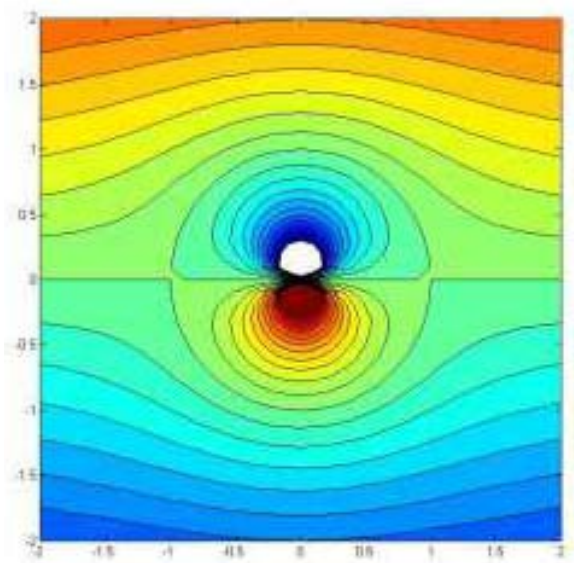

Fig. 8. Velocity contours from Matlab in-built functions
Meshless technique is showing a decent correlation with the vector plots obtained through STAR-CCM+.

\section{CONCLUSIONS AND FUTURE RECOMMENDATIONS}

In this work, Meshfree schemes was developed successfully which creates a cloud of scattered points in the domain and determines the Lagrangian coefficients for each point in and on the domain. Later, the code determines the velocity parameters by implementing the boundary conditions on the basis of potential flow equation. Gauss Seidel approach has been considered for determining the Lagrangian coefficients. Numerical simulation is performed for sample data and the results are validated with the results obtained by FEM. From the above CFD simulations performed, Results and discussions showed that Meshless scheme was able to capture the velocity at each grid point in domain. As in the present thesis, only potential problem is solved, this code can be developed to many applications in engineering problems. As we know, every huge research was initiated with a basic understanding and learning. Therefore, besides the developing of Meshless code in this thesis, the main objective of this thesis is to understand the basic methodology involved in developing a Meshless code. From this simple methodology, one can develop many other Meshless codes which are in need to the engineering simulation world.

\section{REFERENCES}

Aluru, N. R. (2000). A point collocation method based on reproducing kernel Approximations. Internat. J. Numer. Methods Engrg. 47-53.

Atluri, S. N. and T. Zhu (1998). A New Meshless Local Petrov-Galerkin (MLPG) Approach in Computational Mechanics. Comput. Mech. 22, 117- 127.

Atluri, S. N., J. Y. Cho, H. G. Kim, (1999). Analysis of Thin Beams, Using the Meshless Local Petrov-Galerkin Method, with Generalized Moving Least Squares Interpolations. Comput. Mech. 24, 334- 347.

Atluri, S. N., S. Shen, (2002). The Meshless Local Petrov-Galerkin (MLPG) Method. Tech Science Press, Stuttgart.

Atluri, S. N., Sladek, J., Sladek, V., Zhu, T. (2000). The Local Boundary Integral Equation (LBIE) and its Meshless Implementation for Linear Elasticity. Comput. Mech. 25, 180-198.

Babuska, I., Banerjee, U, Osborn, J. E. (2010). Survey of Meshless and generalized finite element methods: A unified approach. Technical Report 02-40, TICAM, the University of Texas at Austin. 
Babuska, I., U. Banerjee, J. E. Osborn (2002). Meshless and generalized finite Element methods - A survey of some major results. In Meshfree Methods for Partial Differential Equations. Springer Verlag, Berlin. Vol. 26.

Francisco, M. B. M. and M. S. Kindelan (2009). A Meshless Solution to the p-Laplace Equation. Computational Methods in Applied Sciences, 978-1402088209.

Gonzalez, D., E. Cueto, and M. Doblare (2009). Towards an Isogeometric Meshless Natural Element Method, Computational Methods in Applied Sciences 978-1402088209.

Han, W. and Meng, X. (2002). Some studies of the reproducing kernel particle method. In Meshfree Methods for Partial Differential Equations, Springer Verlag Vol. 26, Berlin.

Han, W. and X. Meng, (2001). Error analysis of the reproducing kernel particle method. Comp. Methods Appl. Mech. Engrg. 190, 6157- 6181.

Hao, S. and Liu, W. K. (2002). Revisit of Moving Particle Finite Element Method. Proceedings of the Fifth World Congress on Computational Mechanics (WCCM V), Vienna.

Hegen, (1996). Element-free Galerkin Methods in Combination with Finite Element Approaches.
Comp. Methods Appl. Mech. Engrg. 135, 143166.

Jiun-Shyan (JS), C., W. Hu, and H. Y. Hu (2009). Localized Radial Basis Functions with Partition of Unity Properties. Computational Methods in Applied Sciences 978-1402088209.

Lakshmanaprabu, S. K., Nasir, A. W. and Banu U. S. (2017). Design of Centralized Fractional order PI Controller for Two Interacting Conical Frustum Tank Level Process. Journal of Applied Fluid Mechanics 10, 23-32.

Michael, M. and S. De (2009). A Partition of UnityBased Multiscale Method. Computational Methods in Applied Sciences, 978-1402088209.

Mulay, B. B. and B. Mira (2009). Particular Solution of Poisson Problems Using Cardinal Lagrangian Polyharmonic Splines. Computational Methods in Applied Sciences 978-1402088209.

Unther, G. F. C. (1998). A Meshfree Formulation for the Numerical Solution of the Viscous Compressible Navier-Stokes Equations, Dissertation. Northwestern University, Evanston, IL. 